\title{
Robustness studies of oscillation-type density meters with viscoelastic fluids
}

\author{
Andreia Furtado $^{1^{*}}$, Jorge Pereira ${ }^{1}$, Raquel Quendera ${ }^{1}$, Maria Teresa Cidade $^{2}$ \\ ${ }^{1}$ Instituto Português da Qualidade (IPQ), Portugal \\ ${ }^{2}$ Departamento de Ciência dos Materiais e CENIMAT-I3N, Faculdade de Ciências e Tecnologia, \\ Universidade Nova de Lisboa, Caparica, Portugal
}

\begin{abstract}
The oscillation frequencies produced by oscillation-type density meters during the density measurements suffer damping due to the viscosity of Newtonian liquids. The effect of viscoelastic behaviour of non-Newtonian liquids in the damping of these oscillations is still not known. So, 7 viscoelastic liquids were rheologically characterized, resorting to a rheometer, and their density measured in order to provide a deeper insight into the damping effects produced by these types of fluids. To do so, oscillatory data was related with the obtained density deviations. The results of this study will give further insights for the knowledge of the measuring behaviour of these density meters when measuring viscoelastic fluids, one of the scopes of the EMPIR Project 17RPT02-rhoLiq.
\end{abstract}

\section{Introduction}

\subsection{Density of fluids}

Density, $\rho$, is one of the major driving elements in economic transactions with important goods, ranging from fuels to liquid food products. The actual consumption of high-value liquids, such as wine, olive oil and fuel, can be considered of major importance to the European economy [1-3]. Fluids' physical properties such as: density, surface tension, viscosity and elasticity (i.e. viscoelastic behavior due to dissolved gases like carbon dioxide in beverages, suspended particles like in the pulp in a natural juice, etc.), among others, cover a wide spectrum of variation. Additionally, these liquids are handled in a wide range of temperature and pressure. So, the existent methods for density measurement are influenced by one or more of these physical properties as well as by temperature and pressure, meaning that their robustness need to be study. However, currently there are no EURAMET guides on fluids density measurements and the existing international standards $[4,5]$ and reference documents used in Legal Metrology [6, 7] are either outdated or incomplete. Improvements in the quality of fluids density measurements will have a direct impact at industrial sites, leading to more competitive national industries and it will allow a free circulation within the EU market.

*Corresponding author: afurtado@ipq.pt 


\subsubsection{Fluids' density measurements}

Oscillation-type density meters are proven to be very robust, reliable and convenient instruments to measure density of (Newtonian) fluids in a wide density, viscosity and temperature ranges, with an uncertainty better than $0.01 \mathrm{~kg} \mathrm{~m}^{-3}$ when using adequate calibration methods. The working principle of an oscillation-type density meter is based on the law of harmonic oscillation. The viscous component of a liquid during the oscillation causes, on the one hand, a formation of a boundary layer which increases the inertial mass of the resonator and on the other hand a damping of the oscillation due to the wall shear stress acting on the resonator. Consequently, these two parasitic effects leads to a lower resonance frequency of the measuring system and, thus, to an incorrect density indication. The detection of these influences is performed by analyzing the frequency of harmonics of the oscillation. So, these effects can be corrected by means of algorithms obtained during the calibration of the oscillator with viscous samples. However, the effect of the viscoelasticity on non-Newtonian fluids in the damping of these oscillations is still not well-described.

Earlier studies [8] indicated that oscillation of density meter measuring cell may cause modifications in the internal structure and arrangement of the molecules of viscoelastic samples, leading to a non-well described/characterized density deviation trend.

In order to provide a deeper insight into the damping effects produced by these types of fluids when measuring density with an oscillation-type density meter, 7 different viscoelastic fluids were prepared, their mechanical properties were study resorting to a rheometer and their density measured by gravimetric method by using a pycnometer.

The results of this study will give further insights for the knowledge of the measuring behavior of this type of density meters when measuring viscoelastic fluids, one of the scopes of the EMPIR Project 17RPT02-rhoLiq [9]. The knowledge gained during this study will be disseminated in international guides and standards for scientific, applied $[4,5]$ and legal $[6,7]$ metrology and this will address the lack of documentation on this issue.

\subsection{Viscoelasticity of fluids}

Viscoelastic materials are always showing viscous and elastic behavior simultaneously. The viscous portion behaves according to Newton's law and the elastic portion to Hooke's law. So, depending on their rheological behavior, viscoelastic materials may behave as viscoelastic liquids or viscoelastic solids

\subsubsection{Oscillatory tests}

Oscillatory tests, performed with rheometers, are ideal to quantify the "amount" of viscosity and elasticity "hidden" in a material's structure. When performed in the nondestructive regime (meaning that the applied forces are too low to alter a material's microstructure), i.e. in the linear-viscoelastic (LVE) range, oscillatory tests can be used, for instance, to study the shelf-life stability of a material or to investigate different kinds of phase transitions, etc.

Couette flow is frequently used to illustrate shear-driven fluid motion. Couette flow is the flow of a fluid in the space between two surfaces, one of which is moving tangentially relative to the other. The configuration often takes the form of two parallel plates or the gap between two concentric cylinders. The simplest conceptual configuration of a Couette flow finds two infinite, parallel plates separated by a distance $h$ (in $\mathrm{m}$ ), where one plate is stationary and the motion of the other plate causes a shear stress $\tau$ (i.e. a force applied tangentially to the movable plate divided by the area, in $\mathrm{N} \mathrm{m}^{-2}$ or in SI units $\mathrm{Pa}$ ) in the sample, which is placed between the plates, leading to deformation. The deflection path $s$ (in $\mathrm{m}$ ) movable plate, is measured and rheologically evaluated as strain or deformation $\gamma$ (in 
$\%)$. This flow assumes that there is an adhesive force between sample and plates and the produced flow is under laminar regime.

During rheological tests in oscillation mode, the material is exposed to a continuous sinusoidal excitation of either a shear deformation or a shear stress (produced by the rheometer). Depending on the type of excitation, the material will respond with a stress or a deformation (measured by the rheometer). When the amplitude values of the applied stress or deformation signal is low, within LVE range, the response of the sample will also show a sinusoidal shape. Depending on the type of sample, the applied sinusoidal signal and the response signal from the sample will show a phase shift, delta $\delta$, between $0^{\circ}$ and $90^{\circ}$. A phase shift of $0^{\circ}$ indicates that the sample shows no viscous response and is considered purely elastic. Consequently, a phase shift of $90^{\circ}$ implies that a material is behaving as purely viscous with no elastic response.

Storage modulus $\mathrm{G}^{\prime}$, in $\mathrm{Pa}$, is a measure of the deformation energy stored by the sample during the shear process, that will stay completely available after the deformation or a stress is removed and can act as driving force for the reformation process. On the other hand, loss modulus G", in Pa, is a measure of the deformation energy used by the sample during the shear process and is, therefore, lost (i.e. dissipated) for the sample trough the viscous heating. Thus, G' represents the elastic behavior of the material and G', the viscous behavior. The loss (or damping) factor $\tan \delta$, a dimensionless quantity, is calculated as the quotient of the lost (G') and the stored deformation energy (G'), i.e. $\tan \delta=\mathrm{G}^{\prime}$ ' $/ \mathrm{G}^{\prime}$. It therefore reveals the ratio of the viscous and the elastic portion of the viscoelastic deformation behavior. So, for the fluid or liquid state ("sol state") holds $\tan \delta>1$ (since G', $>$ G'), for the gel-like state or solid state holds $\tan \delta<1$ (since G' $>$ G',) and for the sol/gel transition point holds $\tan \delta=1$ (since G' $=$ G' ').

Different measurements become available when an oscillatory deformation or stress is applied to a sample. These measurements include: Oscillatory Amplitude Sweep and Oscillatory Frequency Sweep.

\subsubsection{Balance of materials' viscoelastic behavior}

If the viscous portion of a viscoelastic material is large enough (i.e. $\tan \delta \rightarrow \infty$, viscoelastic liquid behavior), the absorption properties of the material are enhanced, e.g. to damp the effects of mechanical vibrations. The deformation arising from mechanical oscillations is used in the energy-absorbing material components (e.g. rubber buffers for damping of mechanical vibrations). On the other hand, if a material has a too large viscous portion, this can lead to excessive viscous heating, and at the end, to a high destructive degree of deformation. In this case, too much deformation energy would be transformed into heat, more than can be simultaneously stored or transport trough the material outside [10].

\subsubsection{Time-dependent structural decomposition and regeneration}

A material that shows a thixotropic behavior presents a reduction of its internal structural strength under a sufficiently high shear deformation that is followed by a complete structural regeneration in the subsequent period of rest (e.g. dispersions and gels). On other hand, a rheopectic behavior means an increasing of the initial structural strength when performing a sufficiently high shear deformation, which is followed by a complete structural regeneration.

\section{Materials and Methods}

\subsection{Test fluids}

To carry out the study of the influence of the viscoelasticity of the samples on the density measurement result of oscillation-type density meters, 5 aqueous solutions of different compositions were chosen: poly(vinyl alcohol) (PVA), at $0.036 \mathrm{~g} \mathrm{~mL}^{-1}$ and sodium borate 
(Borax) at $0.0036 \mathrm{~g} \mathrm{~mL}^{-1}$; carboxylpolymethylene (Carbomer) solution at $0.15 \mathrm{cg} \mathrm{g}^{-1}$; hydroxyethyl-cellulose at $0.5 \mathrm{cg} \mathrm{g}^{-1}$; starch dispersion at $3 \mathrm{cg} \mathrm{g}^{-1}$ and poly(acrylamide-codiallyldimethylammonium chloride) (P(AAm-co-DADMAC) solution at $5.5 \mathrm{cg} \mathrm{g}^{-1}$. In addition, two commercial beverages (apple juice and grape juice) were also tested. For simplification further references to the test fluids will be given by using the codes attributed in Table 1.

Table 1. Codification of the test fluids (F\#).

\begin{tabular}{|c|l|}
\hline Codification & Fluid \\
\hline F1 & PVA and Borax \\
\hline F2 & Carbomer \\
\hline F3 & Hydroxyethyl-cellulose \\
\hline F4 & Starch dispersion \\
\hline F5 & P(AAm-co-DADMAC) \\
\hline F6 & Apple juice \\
\hline F7 & Grape juice \\
\hline
\end{tabular}

\subsubsection{PVA and Borax solution}

A solution of PVA, at $0.036 \mathrm{~g} \mathrm{~mL}^{-1}$, and Borax at $0.0036 \mathrm{~g} \mathrm{~mL}^{-1}$, was prepared as described below, based on [11].

The PVA solution, at $0.04 \mathrm{~g} \mathrm{~mL}^{-1}$, was prepared by slowly adding $40 \mathrm{~g}$ of PVA (87$90 \%$ hydrolyzed, average molecular mass [30.000-70.000] $\mathrm{g} \mathrm{mol}^{-1}$, CAS: 9002-89-5, Sigma-Aldrich,) to $800 \mathrm{~mL}$ of deionized water preheated to $70^{\circ} \mathrm{C}-80^{\circ} \mathrm{C}$ and stirred until all the polymer was dissolved. It was ensured that the solution did not boil. The mixture was allowed to cool to room temperature and transferred into a $1000 \mathrm{~mL}$ volumetric flask. Deionized water was then added until the mark and the solution was finally mixed by slowly inverting a few times.

The Borax solution, at $0.04 \mathrm{~g} \mathrm{~mL}^{-1}$, was prepared by transferring $4 \mathrm{~g}$ of sodium tetraborate decahydrate (anhydrous, Puriss P.A., ACS reagen, Reag. ISO, buffer substance, $\geq 99.5 \%$, CAS: 1303-96-4, Honeywell, Fluka) into a $100 \mathrm{~mL}$ volumetric flask to which ultrapure water was added, dissolving the borax, by heating, and then cooled to room temperature

The PVA and Borax solutions were finally jointed and mixed in a $2 \mathrm{~L}$ flask.

\subsubsection{Carbopol solution}

A Carbomer solution at $0.15 \mathrm{cg} \mathrm{g}^{-1}$ was prepared as described below based on [12] Carbomer requires a long time to swell properly for use. So, it is necessary to make a pregel before beginning the ultimate batch. Consequently, carbomer, type 940 (CAS: 9003-014, Carbomera, fagron), was weighted and added to a known volume of ultrapure water at room temperature. The sample was left for $24 \mathrm{~h}$ at room temperature. The sample was then stirred for $2 \mathrm{~h}$ at $350 \mathrm{rpm}$ by using a magnetic stirrer, to assure homogeneity and dispersity of carbomer in the aqueous solution.

\subsubsection{Hydroxyethyl-cellulose solution}

A hydroxyethyl-cellulose (Sigma-Aldrich, CAS: 9004-62-0) solution at $0.5 \mathrm{cg} \mathrm{g}^{-1}$ on ultrapure water was prepared by gravimetric method, based on [13]. The sample was stirred for $24 \mathrm{~h}$ at $350 \mathrm{rpm}$, at room temperature, by using a magnetic stirrer.

\subsubsection{Starch solution}

A starch dispersion (from potato soluble, reag. USP, Ph. Eur, for analysis, CAS: 9005-84-9, Panreac Applichem, ITW Reagents), at $3 \mathrm{cg} \mathrm{g}^{-1}$, was prepared by mixing starch with ultrapure water in a flask. The starch dispersion was appropriately stirred for $30 \mathrm{~min}$ at room temperature and then heated in a $90{ }^{\circ} \mathrm{C}$ water bath for $30 \mathrm{~min}$ with constant mild 
agitation using a magnetic stirrer in order to avoid sedimentation and agglomeration (adapted from [14]). Sorbic acid (synthesis grade, CAS: 110-44-1, Scharlau) was added to the solution to work as stabilizer ( $0.3 \mathrm{~g}$ per $1000 \mathrm{~g}$ of solution).

\subsubsection{P(Aam-co-DADMAC) solution}

A poly(acrylamide-co-diallyldimethylammonium chloride) (P(AAm-co-DADMAC) solution $10 \mathrm{cg} \mathrm{g}^{-1}$ in water, CAS: 26590-05-06, Sigma-Aldrich) solution $5.5 \mathrm{cg} \mathrm{g}^{-1}$ in ultrapure water was prepared by weighing. The solution was stirred at $200 \mathrm{rpm}$ with a magnetic stirrer for $24 \mathrm{~h}$.

\subsection{Density measurements}

To perform this study a commercial oscillation-type density meter was used (DMA 5000, Anton Paar). The density indication errors were obtained by calibration of this density meter with Newtonian certified reference liquids (CRM) within the dynamic viscosity, $\eta$, interval from 1 to $795 \mathrm{mPa} \cdot \mathrm{s}$. The reference density values of these CRM were determined by hydrostatic weighing. The density indication errors of the density meter were calculated by the difference between the measured density value and the reference density value [15].

Additionally, the density of the 7 test samples was determined by gravimetric method with the use of a $100 \mathrm{~mL}$ aluminium pycnometer (Erichsen), a mass comparator (Mettler Toledo, PR 2007) and a set of stainless steel standard mass OIML class E2 (Mettler). The tests were performed by substitution weighing method and using the approach described in ISO 2811-1 [16].

The density values of the test fluids obtained by the oscillation-type density meters (i.e., $\rho_{\mathrm{OD} n \mathrm{c}}$ non-viscosity-corrected density value, $\rho_{\mathrm{OD}}$ viscosity-corrected density indication value and $\rho_{\text {OD c }}$ density value corrected with the calibration curve obtained with the Newtonian liquids) were compared against the density results obtained by gravimetric method using a pycnometer $\left(\rho_{\mathrm{PN}}\right)$. The method that the density meters uses to calculate the density correction is not published in detail, thus, a physical model cannot be used for the calculation of viscosity.

Additionally, to test the sensibility of density measurements to samples' time-dependent relaxation/recovery behavior, samples' density were measured at time 0 and at time 5 min, i.e. the same sample was measured twice with a relaxation time interval of $5 \mathrm{~min}$ in between. From these measurements, all the relevant data given by the density meter, i.e. the first and second oscillations periods, $T_{1}$ and $T_{2}$, respectively and the damping indication parameter $Q$, were related. The density meter cell filled with a fluid behaves like a damped harmonic oscillator due to the viscous portion of the sample the $Q$ factor is therefore a ratio between the energy stored (in the oscillating resonator) and the energy dissipated. These results were crossed with the results of the thixotropy tests described in point 2.3.3.

\subsubsection{Uncertainty budget}

The uncertainty of density values obtained in both measuring methods, i.e. with the oscillation-type density meter (1) and with the pycnometer (2), was obtained according to GUM methodology [17], having into account the following major contributions for the uncertainty budget: (1) density meter (resolution, drift and calibration including CRM used), measurements repeatability; (2) calibration of pycnometer volume, balance (resolution, drift and calibration), air buoyancy (and instruments used to measure air temperature, relative humidity and pressure), mass standards used, measurements repeatability, temperature, temperature coefficient of the liquid. In this paper the reported expanded uncertainty, $U$, is stated as the standard measurement uncertainty multiplied by the coverage factor $k=2$, which for a t-distribution corresponds to a coverage probability of approximately $95 \%$. 


\subsection{Rheological determinations}

Complex viscosity $\left|\eta^{*}\right|$, storage modulus, G', and loss modulus, G', dependence on shear strain $\gamma$ and on frequency, $f$, of the 7 viscoelastic samples tested were determined by using a rheometer (HAAKE Mars III, ThermoScientific) using two different measuring geometries: a cone-plate $\left(\mathrm{C} 35 / 2^{\circ} \mathrm{Ti} \mathrm{L}\right)$ for $\mathrm{P}(\mathrm{AAm}-\mathrm{co}-\mathrm{DADMAC})$ and PVA and Borax samples and a concentric-cylinder (CC25 Din Ti) for the remaining samples.

\subsubsection{Oscillatory tests}

Two different oscillation tests were performed in controlled deformation (CD) mode for each sample: (1) amplitude sweep, at constant frequency, $f$, of $1 \mathrm{~Hz}(1.592 \mathrm{~Hz}$ in case of $\mathrm{P}(\mathrm{AAm}-\mathrm{A}$-DADMAC) sample) in the interval of shear strain, $\gamma$, from 0.1 to $100 \%$ (for a total of 16 data points in logarithmic distribution) for the determination of the linear viscoelastic (LVE) range and (2) frequency sweep at a constant strain (deformation) $\gamma$, of $1 \%(0.1 \%$ in case of grape juice sample) inside the LVE range, for the frequency interval, $f$, from 0.1 to $100 \mathrm{~Hz}$ (for a total of 6 data points in logarithmic distribution). Additionally, the amplitude sweep tests gave information regarding the prevalent behaviour of the samples in the LVE range under a low frequency $(1-1.592 \mathrm{~Hz})$, regarding the shear strain applied and to check for the flow stress value, $\tau_{\mathrm{f}}$.

\subsubsection{Data transformation}

Frequency sweeps, ran for a non-destructive deformation range (i.e. in the LVE region), gave information regarding the dependence of the dynamic viscosity, $\eta$, with the oscillation frequency, $f$, up to $100 \mathrm{~Hz}$, the upper limit of the measuring geometries used.

The storage modulus G' $\left(f_{\rho}\right)$ and loss modulus G' $\left(f_{\rho}\right)$ values, at the frequency value $f_{\rho}$ i.e., at the oscillation frequency produced in the oscillation-type density meter during the density measurement of each test sample (usually in the frequency interval of $273-279 \mathrm{~Hz}$ for liquid samples), were estimated by extrapolating the linear regressions of $G^{\prime}(f)$ and $\mathrm{G}^{\prime \prime}(f)$ (eq. 1 and 2, respectively) determined with the experimental data.

$$
\begin{aligned}
& \log \left(\mathrm{G}^{\prime}\right)=a \log (f)+b \\
& \log \left(\mathrm{G}^{\prime}\right)=c \log (f)+\mathrm{d}
\end{aligned}
$$

From the calculated values of $\mathrm{G}^{\prime}\left(f_{\rho}\right)$ and $\mathrm{G}^{\prime}$ ' $\left(f_{\rho}\right)$, viscous portion $\eta^{\prime}\left(f_{\rho}\right)$, i.e. dynamic viscosity, and elastic portion $\eta^{\prime \prime}\left(f_{\rho}\right)$ of samples behavior under shear at frequency $f_{\rho}$, were calculated from equation 3 and 4 , respectively.

$$
\begin{aligned}
& \eta^{\prime}\left(f_{\rho}\right)=\mathrm{G}{ }^{\prime}\left(f_{\rho}\right) / 2 \pi f_{\rho} \\
& \eta^{\prime \prime}\left(f_{\rho}\right)=\mathrm{G}^{\prime}\left(f_{\rho}\right) / 2 \pi f_{\rho}
\end{aligned}
$$

The loss or damping factor, $\tan \delta\left(f_{\rho}\right)$, was calculated as the quotient of the lost $\left(G^{\prime \prime}\left(f_{\rho}\right)\right)$ and the storage modulus $\left(G^{\prime}\left(f_{\rho}\right)\right)$. Thus, this parameter gives the ratio between the viscous and the elastic portion of the viscoelastic deformation.

$$
\tan \delta\left(f_{\rho}\right)=\mathrm{G}^{\prime}\left(f_{\rho}\right) / \mathrm{G}^{\prime}\left(f_{\rho}\right)
$$

If $\tan \delta>1$ (G" > G') the sample is in fluid or liquid state ("sol state"), i.e., showing a behavior of a viscoelastic liquid, for $\tan \delta<1$ ( $\mathrm{G}^{\prime}>\mathrm{G}$ ") the sample is in a gel-like or solid state, i.e., showing a behavior of viscoelastic solid, and for $\tan \delta=1 \mathrm{a} \mathrm{sol} /$ gel transition will be met, i.e., having a viscoelastic behavior showing 50/50 ratio of the viscous and elastic portions. 


\subsubsection{Thixotropic behaviour investigation tests}

The thixotropic behavior of the tested samples was investigated through shear rate ramps loops (upwards and downwards) measurements. Each one of these test was performed in controlled rate $(\mathrm{CR})$ mode , and was divided in 3 parts: (1) rotational ramp for increasing shear rate $\dot{\gamma}$ values from $0.0001 \mathrm{~s}^{-1}$ to $100 \mathrm{~s}^{-1}$ (during $100 \mathrm{~s}$ for a total of 100 data points in linear distribution); (2) rotation at constant shear rate $\dot{\gamma}$ value of $100 \mathrm{~s}^{1}$ (during $30 \mathrm{~s}$ for a total of 30 data points); and (3) rotational ramp for decreasing shear rate $\dot{\gamma}$ values from $100 \mathrm{~s}^{-1}$ to $0.0001 \mathrm{~s}^{-1}$ (during $100 \mathrm{~s}$ for a total of 100 data points in linear distribution).

Flow curves $(\tau(\dot{\gamma}))$ were plotted for each sample and the hysteresis area of each one of the tests parts (i.e., upward shear rate ramp (A1); constant shear rate (A2) and downward shear rate ramp (A3)) were determined by rheometer's software (HAAKE RheoWin Datamanager $\left.{ }^{\circledR}\right)$.

\section{Results}

\subsection{Comparison of the density results}

To study the influence of samples' viscoelasticity on the density measurement results of an oscillation-type density meter, 7 different samples were prepared and rheologically characterized.

Because it is a static method of measuring the density of liquids, pycnometry was chosen to be used in comparison with the ones obtained with the oscillation-type density meter. As a static method it is expected that viscoelastic properties of samples would not influence density measurement results, as it might do in oscillation-type density meters. However, phenomena related to these properties were observed during the filling of the pycnometer which may have influenced the measurement results, and which cannot be easily accounted for.

The compilation of the density results of the 7 test fluids obtained at $20{ }^{\circ} \mathrm{C}$ and ambient pressure by these two density measurement methodologies (oscillation-type density meter $\left(\rho_{\mathrm{OD}}\right)$ and pycnometer $\left.\left(\rho_{\mathrm{PN}}\right)\right)$ are given in Table 2. A maximum relative expanded uncertainty of $0.0053 \%$ was obtained for the density values obtained from the oscillation-type density meter ( $\rho_{\mathrm{OD} \mathrm{nc}}, \rho_{\mathrm{OD}}$ and $\rho_{\mathrm{OD}}$ values and the maximum uncertainty obtained for the pycnometric method was $0.010 \%$ (Table 2 ).

Table 2. Compilation of the density results of the 7 test fluids obtained at $20{ }^{\circ} \mathrm{C}$ and ambient pressure by using an oscillation-type density meter $\left(\rho_{\mathrm{OD}}\right)$ and a pycnometer $\left(\rho_{\mathrm{PN}}\right)$.

\begin{tabular}{|c|c|c|c|c|c|c|}
\hline \multirow{2}{*}{ F\# } & \multicolumn{3}{|c|}{ Oscillation-type density meter (OD) } & \multicolumn{2}{c|}{ Pycnometer (PN) } \\
\cline { 2 - 7 } & $\rho_{\mathrm{OD} \mathrm{nc}}$ & $\rho_{\mathrm{OD} c}$ & $\rho_{\mathrm{OD} c}^{\prime}$ & $U \rho_{\mathrm{OD}}$ & $\rho_{\mathrm{PN}}$ & $U \rho_{\mathrm{PN}}$ \\
\cline { 2 - 7 } & \multicolumn{5}{|c|}{$\left(\mathrm{kg} \mathrm{m}^{-3}\right)$} \\
\hline F1 & 1008.319 & 1008.183 & 1008.542 & 0.054 & 1007.87 & 0.10 \\
\hline F2 & 998.705 & 998.692 & 998.701 & 0.021 & 998.41 & 0.10 \\
\hline F3 & 999.801 & 999.753 & 999.905 & 0.054 & 999.23 & 0.10 \\
\hline F4 & 1008.335 & 1008.324 & 1008.335 & 0.020 & 1008.21 & 0.10 \\
\hline F5 & 1009.954 & 1009.727 & 1010.308 & 0.055 & 1011.52 & 0.10 \\
\hline F6 & 1049.012 & 1048.996 & 1049.005 & 0.021 & 1049.40 & 0.11 \\
\hline F7 & 1068.167 & 1068.164 & 1068.161 & 0.021 & 1067.55 & 0.11 \\
\hline
\end{tabular}

Notes: F\# - codification of the test fluids according to Table $1 ; \rho_{\mathrm{OD} n c}$ - non-viscosity-corrected density value, $\rho_{\mathrm{OD}}$ - viscosity-corrected density value and $\rho_{\mathrm{OD}}^{\prime}$ - density value corrected with the calibration curve obtained with the Newtonian liquids; $U$ - expanded uncertainty stated as the standard measurement uncertainty multiplied by the coverage factor $k=2$, which for a $t$ distribution corresponds to a coverage probability of approximately $95 \%$, obtained according to GUM methodology [17]. 
Table 3 summarizes the density deviations, $\delta \rho$, of the results obtained by the oscillation-type density meter $\left(\delta \rho\left({ }_{\mathrm{OD}} ;{ }_{P N}\right)\right)$ and the results obtained by gravimetric method using a pycnometer $\left(\rho_{\mathrm{PN}}\right)$ of the 7 test fluids. The expanded uncertainties of the density deviations values, $\delta \rho\left({ }_{\mathrm{OD}} ;{ }_{P N}\right)$, were calculated by equation 5 .

$$
U_{\delta \rho}=\sqrt{\left(u \rho_{\mathrm{OD} \mathrm{nc}}\right)^{2}+\left(u \rho_{\mathrm{PN}}\right)^{2}}
$$

Table 3. Summary of the density deviations, $\delta \rho$, of the results obtained by the oscillation-type density meter $\left(\rho_{\mathrm{OD}}\right)$ and the results obtained by gravimetric method using a pycnometer $\left(\rho_{\mathrm{PN}}\right)$ of the 7 test fluids.

\begin{tabular}{|c|c|c|c|c|}
\hline \multirow{2}{*}{ F\# } & \multicolumn{4}{|c|}{$\delta \rho\left(\mathrm{OD} ;{ }_{P N}\right)$} \\
\cline { 2 - 5 } & $\begin{array}{c}\rho_{\mathrm{OD} \mathrm{nc}}-\rho_{\mathrm{PN}} \\
\%\end{array}$ & $\begin{array}{c}\rho_{\mathrm{OD}}-\rho_{\mathrm{PN}} \\
\%\end{array}$ & $\begin{array}{c}\rho_{\mathrm{OD} \mathrm{c}}^{\prime}-\rho_{\mathrm{PN}} \\
\%\end{array}$ & $\begin{array}{c}U_{\delta \rho} \\
\%\end{array}$ \\
\hline F1 & 0.045 & 0.031 & 0.067 & 0.011 \\
\hline F2 & 0.029 & 0.028 & 0.029 & 0.010 \\
\hline F3 & 0.057 & 0.052 & 0.067 & 0.011 \\
\hline F4 & 0.012 & 0.011 & 0.012 & 0.010 \\
\hline F5 & -0.155 & -0.178 & -0.120 & 0.011 \\
\hline F6 & -0.037 & -0.038 & -0.038 & 0.010 \\
\hline F7 & 0.058 & 0.058 & 0.057 & 0.010 \\
\hline
\end{tabular}

Notes: F\# - codification of the test fluids according to Table 1; $\rho_{\mathrm{OD} \text { nc }}$ - non-viscosity-corrected density value, $\rho_{\mathrm{OD} c}$ - viscosity-corrected density value and $\rho_{\mathrm{OD} c}-$ density value corrected with the calibration curve obtained with the Newtonian liquids; $U_{\delta \rho}$ - relative expanded uncertainty of the density deviations values, $\delta \rho\left({ }_{\mathrm{OD}} ;{ }_{P N}\right)$ calculated by equation 5 , stated as the relative standard measurement uncertainty multiplied by the coverage factor $k=2$, which for a $t$-distribution corresponds to a coverage probability of approximately $95 \%$, obtained according to GUM methodology [17].

Table 3 shows that even the density results corrected with the calibration curve obtained with the Newtonian liquids ( $\rho_{\text {OD c }}^{\prime}$ ) produce significant density deviations, up to a maximum value of $\sim 0.12 \%\left(\delta \rho\left(\mathrm{OD} ;{ }_{P N}\right)_{\max }\right)$, when measuring viscoelastic samples, the, i.e. for all samples the density deviation obtained from the density value obtained with the pycnometer is larger than the expanded uncertainty of the density deviations values $\left(U_{\delta \rho}\right)$ (from 0.010 to $0.011 \%$ ). Thus, the obtained density deviations obtained for the viscoelastic samples are 1 to 10 orders higher than the uncertainty of the relative expanded uncertainty of the density deviations values, $\delta \rho\left(\mathrm{OD} ;{ }_{P N}\right)$. The same maximum density deviation value was obtained for the viscosity-corrected density indication value $\left(\delta \rho\left(\mathrm{OD}_{\mathrm{c}} ;{ }_{P N}\right)_{\max }\right)$ given by the density meter. Since these two set of results were corrected according to the damping produce by Newtonian materials it does not take into account the possible effect of sample's elasticity at such high frequency.

\subsection{Relation with samples' mechanical properties}

The oscillation frequency $f_{\rho}$ values (in $\mathrm{Hz}$ ) of each sample during the density measurements with the oscillation-type density meter were calculated by the inverse of the period of the first oscillation $\left(T_{1}\right)$ measured by the density meter. As the tested density meter performs corrections of density values according to the damping in the oscillation period produced by the viscous part of the samples, the estimated value for the viscosity, $\eta_{\text {est. }}$, was also calculated using the approach applied in earlier studies [8]. However, when testing viscoelastic samples, as the results in Table 4 show, the same approach [8] is no longer valid to estimate the viscous portion of a sample. All the viscoelastic samples tested showed a damping factor, $\tan \delta<1\left(\mathrm{G}^{\prime}>\mathrm{G}^{\prime}\right.$ '), indicating that all the tested samples present a 
predominantly solid-like character, i.e., they behave as viscoelastic solids at the frequency $f_{\rho}$ produced in the density meter (Table 4 ).

Table 4. Oscillation frequencies, $f_{\rho}$ and dynamic viscosity $\eta_{\text {est. }}$ values estimated with the oscillationtype density meter and viscous, $\eta^{\prime}\left(f_{\rho}\right)$, elastic, $\eta^{\prime \prime}\left(f_{\rho}\right)$ complex, $\left|\eta^{*}\left(f_{\rho}\right)\right|$ viscosities and loss factor $\tan \delta\left(f_{\rho}\right)$, for $f_{\rho}$, determined by extrapolation of the data obtained in frequency sweep tests performed with the rheometer

\begin{tabular}{|c|c|c|c|c|c|c|c|}
\hline \multirow{2}{*}{ F\# } & \multicolumn{3}{|c|}{ Density meter } & \multicolumn{4}{c|}{ Rheometer } \\
\cline { 2 - 8 } & $\begin{array}{c}f_{\rho} \\
(\mathrm{Hz})\end{array}$ & $\begin{array}{c}Q \\
(1)\end{array}$ & $\begin{array}{c}\eta_{\text {est. }} \\
(\mathrm{mPa} \cdot \mathrm{s})\end{array}$ & $\begin{array}{c}\eta^{\prime}\left(f_{\rho}\right) \\
(\mathrm{mPa} \cdot \mathrm{s})\end{array}$ & $\begin{array}{c}\eta^{\prime \prime}\left(f_{\rho}\right) \\
(\mathrm{mPa} \cdot \mathrm{s})\end{array}$ & $\begin{array}{c}\left|\eta^{*}\left(f_{\rho}\right)\right| \\
(\mathrm{mPa} \cdot \mathrm{s})\end{array}$ & $\begin{array}{c}\text { Tan } \delta\left(f_{\rho}\right) \\
(\mathrm{mPa} \cdot \mathrm{s})\end{array}$ \\
\hline F1 & 277.92 & 2.780 & 39.10 & 0.09 & 170 & 169.7 & 0.0006 \\
\hline F2 & 278.58 & 2.712 & $\leq 5.94$ & 8.20 & 5088 & 5088.1 & 0.0016 \\
\hline F3 & 278.50 & 2.774 & 13.06 & 0.81 & 2456 & 2456.2 & 0.0003 \\
\hline F4 & 277.92 & 2.780 & $\leq 5.94$ & 20.29 & 6164 & 6164.0 & 0.0033 \\
\hline F5 & 277.81 & 2.781 & 65.45 & 0.07 & 1088 & 1087.5 & 0.0001 \\
\hline F6 & 275.17 & 2.689 & $\leq 5.94$ & 5.59 & 21784 & 21784.4 & 0.0003 \\
\hline F7 & 273.91 & 2.821 & $\leq 5.94$ & 2.21 & 951 & 951.0 & 0.0023 \\
\hline
\end{tabular}

Legend: $f_{\rho}$ - oscillation frequency produced in the oscillation-type density meter (DMA 5000, Anton Paar) during the density measurement $(\mathrm{Hz}) ; Q$ - oscillation damping factor; Relative expanded uncertainty of viscosity estimation: $U^{\prime} \eta_{\text {est. }}=18 \%$ [8]; $U^{\prime} \eta^{\prime}\left(f_{\rho}\right)=U^{\prime} \eta^{\prime \prime}\left(f_{\rho}\right)=10-20 \%$.

Also to be noticed that, in general, the difference between the dynamic viscosity values estimated with the oscillation-type density meter, $\eta_{\text {est. }}$, by using the approach used with Newtonian fluids [8], and the viscous $\eta^{\prime}\left(f_{\rho}\right)$ portion of the complex viscosity obtained by extrapolation of the data obtained in frequency sweep tests performed with the rheometer, is higher than the relative expanded uncertainty of viscosity estimations $\left(U^{\prime} \eta_{\text {est. }}=18 \%\right.$ [8]; U' $\left.\eta^{\prime}\left(f_{\rho}\right)=U^{\prime} \eta^{\prime}\left(f_{\rho}\right)=10-20 \%\right)$ meaning that the approach used with Newtonian fluids [8] to estimate samples' viscosity values $\eta_{\text {est }}$, is not suitable for nonNewtonian fluids (Table 4).

Table 5. Summary of the viscoelastic prevalent behavior of the 7 tested samples in the linear viscoelastic range (in amplitude sweep tests at low frequency) and under high frequencies (in frequency sweep tests), performed at $20^{\circ} \mathrm{C}$

\begin{tabular}{|c|c|c|}
\hline \multirow{2}{*}{ F\# } & \multicolumn{2}{|c|}{ Oscillatory tests } \\
\cline { 2 - 2 } & $\begin{array}{c}\text { Amplitude sweep }(f=1 \mathrm{~Hz}) \\
\text { Viscoelastic behavior in the LVE range }\end{array}$ & $\begin{array}{c}\text { Frequency sweep }(f \sim 273-279 \mathrm{~Hz}) \\
\text { Viscoelastic behavior predicted for } f_{\rho}\end{array}$ \\
\hline F1 & G'> G', liquid-like & \\
\hline F2 & G' $>$ G', solid/gel-like & \multirow{2}{*}{$\mathrm{G}^{\prime}>\mathrm{G}^{\prime \prime}$, solid/gel-like } \\
\hline F3 & G', $>$ G', liquid-like & \\
\hline F4 & & \\
\hline F5 & G' $>$ G', solid/gel-like & \\
\hline F6 & F7 &
\end{tabular}

Legend: for the fluid F5 the amplitude sweep test was performed at a constant frequency of $1.592 \mathrm{~Hz}$.

In terms of viscoelastic behaviour one can group the test samples in three different groups, according to the results of the amplitude sweep tests: (1) samples F1, F3 and F5 (PVA and Borax, hydroxyethyl-cellulose and P(AAm-co-DADMAC) solutions) exhibited liquid-like behaviour ( $\mathrm{G}^{\prime}$ ' $>\mathrm{G}$ ') in the entire shear strain, $\gamma$, interval tested (i.e., from 0.1 to $100 \%$ ), therefore acting like viscoelastic liquids; (2) samples F2, F4 and F7 (carbomer 
solution, starch solutions and grape juice samples) exhibited a solid-like character $\left(G^{\prime}>G^{\prime}\right.$ ') in the LVE range (Table 5), with a flow stress, $\tau_{f}\left(G^{\prime}=G^{\prime \prime}\right)$ between 1.8 $2.7 \mathrm{~m} \mathrm{~Pa}, 10.6$ - $16.5 \mathrm{~m} \mathrm{~Pa}$, and $1.7-2.8 \mathrm{~m} \mathrm{~Pa}$, respectively; (3) sample F6 (apple juice sample) showed a gel-like character $\left(G^{\prime}>G^{\prime}\right.$ ') in the entire shear strain, $\gamma$, interval tested (Table 5).

On the other hand, the rheological results, at the same frequency produced by the density meter, $f_{\rho}$, obtained by extrapolation of the frequency sweep test results, showed that all tested samples showed solid-like behaviour (G', $>$ G') (Table 5), with a damping factor between 0.0006 and 0.0033 (Table 4). As the damping factor $\tan \delta\left(f_{\rho}\right)$ is the quotient of lost G' (viscous portion) and storage modulus G' (elastic portion) (eq. 4), small values of $\tan \delta$, i.e. $<1$, are related with solid like behavior. Additionally, from frequency sweep tests, it was possible to realize that at low frequencies, F1, F2, F3, F4, F6 and F7 samples (PVA and Borax, carbomer, hydroxyethyl-cellulose, starch solutions and grape juice samples) presented a liquid-like behaviour (G'> G'). F5 sample (P(AAm-co-DADMAC) solution was the only one showing a gel-like character $\left(G^{\prime}>G^{\prime \prime}\right)$ at low frequencies and presenting two crossover points $\left(\mathrm{G}^{\prime}{ }^{\prime}=\mathrm{G}^{\prime}\right)$ at frequencies $f$ around $19.0 \mathrm{~Hz}$ and $59.3 \mathrm{~Hz}$.

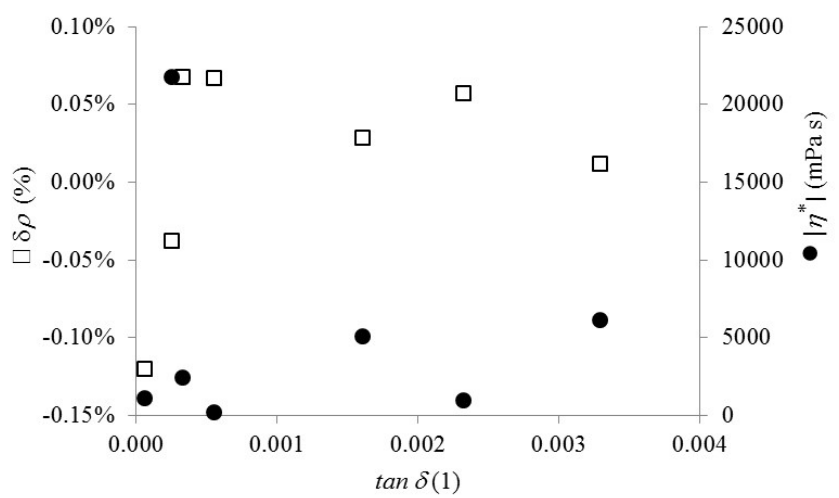

Fig. 1. Graphic representation of the density deviation $\delta \rho$ (i.e., $\rho_{\mathrm{OD}}^{\prime}-\rho_{\mathrm{PN}}$ ) (white squares) and the complex viscosity $\left|\eta^{*}\right|$ (black filled circles) of the 7 viscoelastic samples, at $20^{\circ} \mathrm{C}$, against the damping values $\tan \delta$ (Table 4 ) of the samples for frequency $f_{\rho}$.

At this point, the major concern of this investigation is to check if there is any causal relation between samples' viscoelastic character and density deviations $\delta \rho$ obtained by the density meter. For this, the density deviation $\delta \rho$ and samples' complex viscosity $\left|\eta^{*}\left(f_{\rho}\right)\right|$ were both plotted against samples' loss (damping) values $\tan \delta$, determined for frequency $f_{\rho}$ in frequency sweep tests with the rheometer, in Figure 1. As previously described, if the viscous portion of a viscoelastic material is large enough (i.e., $\tan \delta \rightarrow \infty$, viscoelastic liquid behavior) its absorption properties are enhanced causing damping effects on mechanical vibrations. However, all the tested samples presented solid-like (i.e., $\tan \delta<1$ as G' $>$ G' ') for the oscillation frequency $f_{\rho}$ produced in the density meter measuring cell during density measurements.

However, in contradiction with what was expected, for samples presenting low values of $\tan \delta$ (i.e., $\tan \delta \rightarrow 0$ ) and lower values of viscous $\eta^{\prime}\left(f_{\rho}\right)$ the density deviations $\delta \rho$ are higher (Figure 1). The highest density deviation was obtained for the P(AAm-coDADMAC) sample $(\delta \rho=-0.12 \%$ and $\tan \delta=0.0001)$.

As previously described, the measuring cell of the density meter when filled with the fluid under test will act as a damped harmonic oscillator due to the viscous portion of the sample, the $Q$ factor (or oscillation damping factor), is therefore a ratio between the energy 
stored (in the oscillating resonator) and the energy dissipated during the oscillation by damping processes (in this case shear friction and shear heating). In this sense, it was expected that $Q$ factor decreased for increasing viscosities in an exponential trend like describe in earlier studies [8] with Newtonian fluids (Fig. 2).

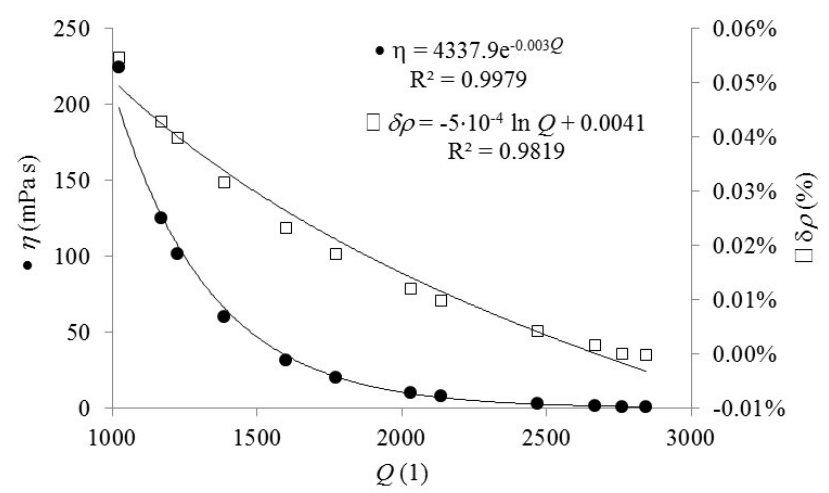

Fig. 2. Curves of the data obtained in [8] with Newtonian liquids by using oscillation-type density meters (DMA 5000M, Anton Paar). Dynamic viscosity $\eta$ (black filled circles), at $20^{\circ} \mathrm{C}$ and $23{ }^{\circ} \mathrm{C}$, and relative density deviation $\delta \rho$ (i.e., $\left.\rho_{\mathrm{OD} \mathrm{c}}^{\prime}-\rho_{\mathrm{PN}}\right)$ (white squares), both against $Q$ factor.

For viscoelastic samples, and when considering $Q$ factor in function of complex viscosity $\left|\eta^{*}\right|$ (i.e., $Q\left(\left|\eta^{*}\right|\right)$ ) in general the trend is the same as the one obtain for Newtonian liquids, i.e. high values of $\left|\eta^{*}\right|$ produce lower values of $Q$, though, density deviations $\delta \rho$ do not fit the expected trend (black filled circles in Fig. 3). Taking a deeper look into the trend of $Q$ against the viscous $\eta^{\prime}$ portion of the complex viscosity $\left|\eta^{*}\right|$, it can be seen that the same trend is no longer existing (black filled circles in Fig. 4). In theory, it was expected that more viscous samples (i.e., with high viscosity values $\left(\eta^{\prime}\right)$ ) would originate lower $Q$ factor values.

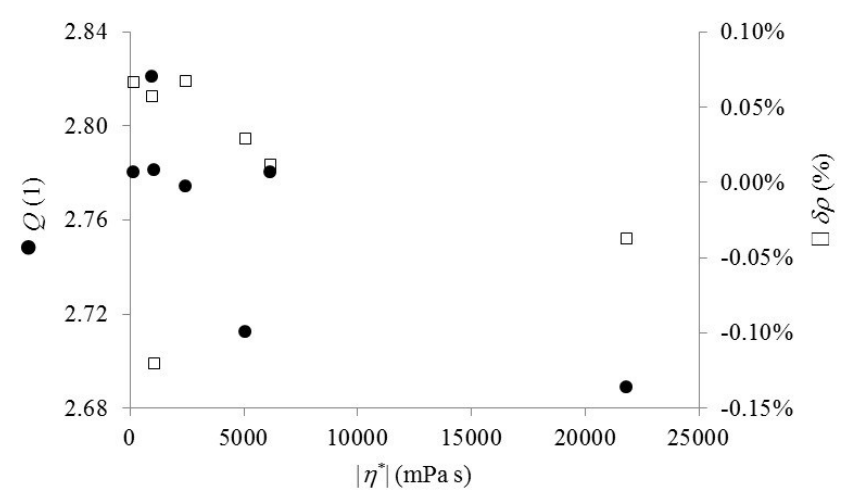

Fig. 3. Graphic representation of oscillation damping values $Q$ (black filled circles) (Table 4 ) and the the relative density deviation $\delta \rho$ (i.e., $\rho_{\mathrm{OD} \mathrm{c}}^{\prime}-\rho_{\mathrm{PN}}$ ) (white squares) (Table 3 ) of the 7 viscoelastic samples, at $20^{\circ} \mathrm{C}$ (Table 4), against samples' complex viscosity $\left|\eta^{*}\right|$ (Table 4) determined for frequency $f_{\rho}$ in frequency sweep tests. 


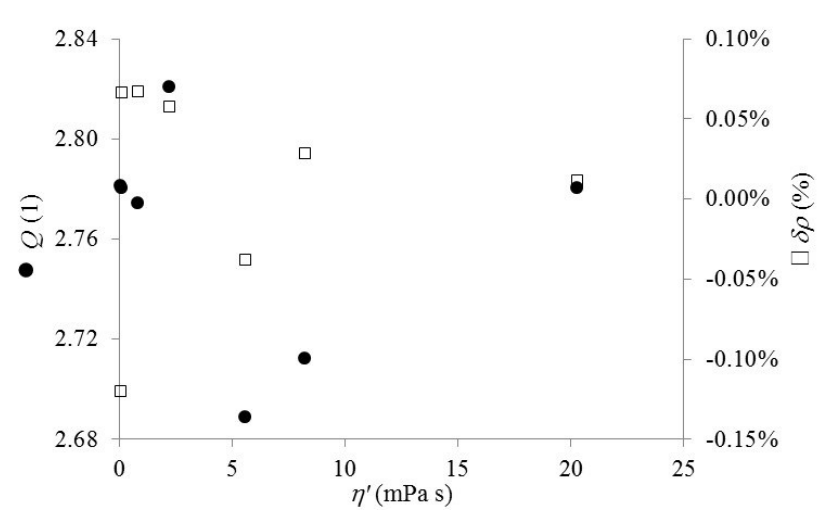

Fig.4. Graphic representation of oscillation damping values $Q$ (black filled circles) (Table 4) and the the relative density deviation $\delta \rho$ (i.e., $\rho_{\mathrm{OD}}^{\prime}-\rho_{\mathrm{PN}}$ ) (white squares) (Table 3 ) of the 7 viscoelastic samples, at $20^{\circ} \mathrm{C}$ (Table 4), against samples' viscous $\eta^{\prime}$ portion of the complex viscosity $\left|\eta^{*}\right|$ (Table 4) determined for frequency $f_{\rho}$ in frequency sweep tests.

Figure 5 summarize the loss factor values $\tan \delta$ (Table 4) of the 7 viscoelastic samples determined for the frequency $f_{\rho}$ in frequency sweep tests against the oscillation damping values $Q$ (Table 4). As the damping oscillation factor $Q$ gives an indication of the ratio of the energy stored (i.e. elastic portion $\eta^{\prime}$, , or storage modulus G') by the energy dissipated (i.e. viscous portion $\eta^{\prime}$, or loss modulus G',) during the oscillation process, it was expected that $Q$ vary inversely with the materials loss factor $\tan \delta$ (resulting from $\mathrm{G}^{\prime \prime} / \mathrm{G}^{\prime}$ or $\eta^{\prime} / \eta^{\prime}$ ), however this is not the case as observed in Fig. 5.

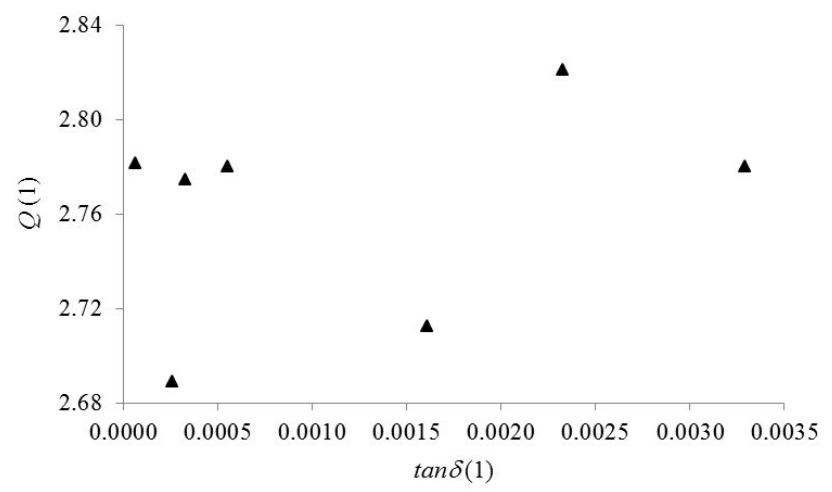

Fig. 5. Graphic representation of oscillation damping values $Q$ against the loss factor $\tan \delta$ of the 7 viscoelastic samples determined for the frequency $f_{\rho}$ in frequency sweep tests (Table 4 ).

\subsection{Relation with samples' time-dependent relaxation/recovery behaviour}

The sensibility of density measurements results to samples' time-dependent relaxation/recovery behavior was tested by measuring the same sample at two different relaxation times, i.e., time 0 and after $\sim 5 \mathrm{~min}$. From these measurements, all the relevant data given by the density meter, i.e. the first and second oscillations periods, $T_{1}$ and $T_{2}$, respectively and the damping indication parameter $Q$, were related (Table 6). These results were also crossed with the results of the thixotropy tests (Table 7). 
Table 6. Summary of sensibility of density measurements results to the 7 test samples' timedependent relaxation/recovery behavior in terms of variation of: density indication $\Delta \rho_{\mathrm{OD}}$; first and second oscillations periods, $T_{1}$ and $T_{2}$ and damping indication parameter $Q$.

\begin{tabular}{|c|c|c|c|c|c|c|}
\hline \multirow{2}{*}{ F\# } & \multirow{2}{*}{$\begin{array}{l}\Delta t \\
\text { (s) }\end{array}$} & $\Delta \rho_{\mathrm{OD} \mathrm{c}}$ & $\Delta \rho_{\mathrm{OD} \mathrm{nc}}$ & $\Delta T_{1}$ & $\Delta T_{2}$ & $\Delta Q$ \\
\hline & & \multicolumn{5}{|c|}{$(\%)$} \\
\hline F1 & 427 & 0.0002 & 0.0001 & 0.03 & 0.2 & 4 \\
\hline F2 & 353 & -0.0004 & -0.0003 & 0 & 16.7 & 5 \\
\hline F3 & 373 & -0.0002 & -0.0002 & 0 & 4.1 & 4 \\
\hline F4 & 507 & -0.0004 & -0.0004 & 0 & 4.2 & 4 \\
\hline F5 & 449 & -0.0002 & -0.0001 & 0 & 0.0 & 0 \\
\hline F6 & 357 & 0.0002 & 0.0006 & 0.14 & 11.8 & 0 \\
\hline F7 & 341 & 0.0005 & 0.0006 & 0.18 & 0.4 & 0 \\
\hline
\end{tabular}

Legend: $\Delta$ means the difference between the values measured at time $\sim 5 \mathrm{~min}$ and time $0 ; \rho_{\mathrm{OD} \mathrm{nc}}-$ nonviscosity-corrected density value, $\rho_{\mathrm{OD}}$ - viscosity-corrected density value.

As was described, samples for which a positive value of resulting hysteresis area (i.e., Diff. in Table 7) was obtained are thixotropic, and those with a negative value of hysteresis area are rheopectic. So, according to Table 7, all the samples tested, with exception of F5 (i.e., P(AAm-co-DADMAC) solution), showed a thixotropic behavior, i.e. presented a reduction of its internal structural strength when subjected to a high-shear deformation, that is followed by a complete structural regeneration in the subsequent period of rest. This type of behavior is common for dispersions and gel samples.

Table 7. Summary of the rotational tests results performed to characterize the thixotropic behavior of the 7 test fluids, at $20^{\circ} \mathrm{C}$

\begin{tabular}{|c|c|c|c|c|}
\hline \multirow{2}{*}{ F\# } & \multicolumn{4}{|c|}{ Rotational tests } \\
\cline { 2 - 5 } & \multicolumn{4}{|c|}{ Thyxotropy test } \\
\cline { 2 - 5 } & Diff $^{*}$ & $\mathrm{~A}_{1}$ & $\mathrm{~A}_{2}$ & $\mathrm{~A}_{3}$ \\
\cline { 2 - 5 } & $\%$ & 1994000 & 139700 & 1739000 \\
\hline F1 & 21 & 15840 & 3.802 & 10520 \\
\hline F2 & 34 & 179500 & 30.59 & 8948 \\
\hline F3 & 3 & 14250 & 2.565 & 2327000 \\
\hline F4 & 37 & 2311000 & 569.4 & 5922 \\
\hline F5 & -1 & 11220 & 3.329 & 6851 \\
\hline F6 & 47 & 12150 & 8.036 & \\
\hline F7 & 44 & & \multicolumn{3}{|c|}{} \\
\hline
\end{tabular}

Legend: ${ }^{*}$ Resulting hysteresis area: Diff $=\left[\left(\mathrm{A}_{1}+\mathrm{A}_{2}-\mathrm{A}_{3}\right) /\left(\mathrm{A}_{1}+\mathrm{A}_{2}-\mathrm{A}_{3}\right)\right] \cdot 100$; Hysteresis areas $\mathrm{A}_{1}, \mathrm{~A}_{2}$ and $A_{3}$ obtained in the tests for the upward shear rate ramp $\left(A_{1}\right)$; constant shear rate $\left(A_{2}\right)$ and downward shear rate ramp. The hysteresis areas values were integrated with rheometer's software (HAAKE RheoWin Datamanager $\left.{ }^{\circledR}\right)$.

The relation between the density deviations $\left(\Delta \rho_{\mathrm{OD}}\right.$ and $\left.\Delta \rho_{\mathrm{OD} n \mathrm{c}}\right)$ (Table 6) and the hysteresis area (Table 7) obtained in the thixotropic behaviour investigation tests is shown in Figure 6. Even if not possible to determine a casual relation between these two quantities, they seem to be more related then the first and second oscillations periods, $T_{1}$ and $T_{2}$ and damping indication parameter $Q$. 


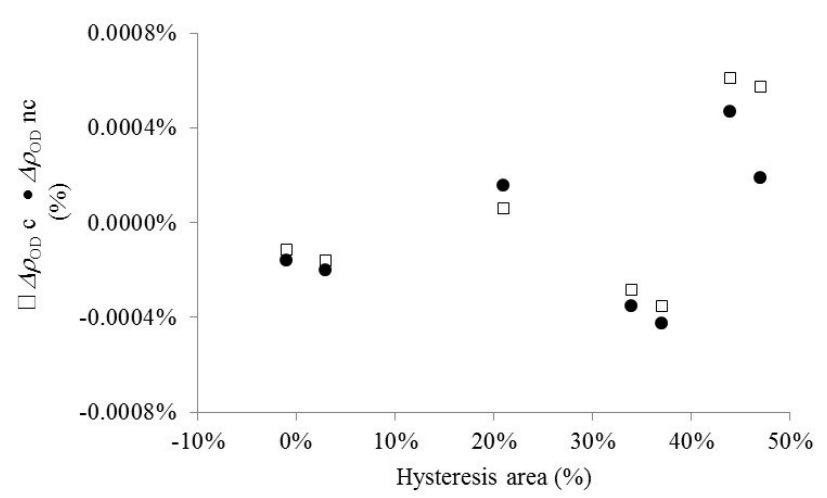

Fig. 6. Graphic representation of relation between density deviations, $\Delta \rho_{\mathrm{OD}}$ (white squares) and $\Delta \rho_{\mathrm{OD} \mathrm{nc}}$ (black filled circles), both in \%, obtained by an oscillation-type density meter (DMA 5000, Anton Paar) when measuring the density of the 7 viscoelastic samples, and the hysteresis area obtained in time-dependent relaxation/recovery behaviour investigation tests performed at $20^{\circ} \mathrm{C}$.

\section{Conclusions}

Oscillation-type density meters have shown to be convenient instruments to measure the density of Newtonian liquids in a wide range of density, viscosity and temperature, with an expanded uncertainty from $0.01-0.03 \mathrm{~kg} \mathrm{~m}^{-3}$, by using a proper calibration curve, since the deviations due to viscosity may lead to a maximum density deviation of $0.62 \mathrm{~kg} \mathrm{~m}^{-3}$ in the viscosity interval up to $795 \mathrm{mPa} \mathrm{s}[8]$.

This study showed that the knowledge of samples' viscoelasticity effect on density measurements results using this kind of density meters is limited by the uncertainty of the pycnometer method $0.010 \%$, since these density meters are able to produce density results with much lower relative uncertainty $(\sim 0.0053 \%)$. So, alternative method needs to be develop/improved as alternative to pycnometer method. An adapted hydrostatic method could be a good approach to be explored in further studies.

As already suspected in earlier studies [8], these results are other indication that oscillation of density meter cell, during density measurements, can cause modifications in the internal structure and arrangement of the molecules of the viscoelastic samples, leading to a non-well described density deviation trend, which may be essentially due to the elastic portion of the viscoelastic behavior of these samples. All the viscoelastic samples tested showed a damping factor, $\tan \delta<1$ (G'> G'), indicating that all the tested samples are in a solid-like state, i.e., showing a behaviour of viscoelastic solids at the frequency $f_{\rho}$ produced in the density meter even when they are in liquid-like state in lower frequencies regimes (PVA and Borax, hydroxyethyl-cellulose, P(AAm-co-DADMAC) solutions and starch dispersion).

The frequency/temperature shift (FTS) method allows to extent various rheological parameters beyond the actual frequency range measured, within which no measuring data are available [10, 18]. And this could be a good alternative to the extrapolation methodology used in this paper to estimate the storage modulus $\mathrm{G}^{\prime}\left(f_{\rho}\right)$ and loss modulus and G' $\left(f_{\rho}\right)$ values, at the frequency value $f_{\rho}$ i.e., oscillation frequency produced in the oscillation-type density meter during density measurements (usually in the frequency interval of 273-279 Hz for liquids samples), gave information regarding the dependence of the dynamic viscosity, $\eta$ ', with the oscillation frequency, f, up to $100 \mathrm{~Hz}$, the upper limit of the measuring geometries used. However, FTS method is only applied to thermorheologically simple materials, not the case of dispersions and gels. For this reason, and since the mechanical characterization and the viscoelastic properties of fluids under such a 
high frequency value is not feasible by means of rheometry due to instrumental limitations, other methodologies might be use, e.g. by indirect method with Dynamic Mechanic Analysis (DMA) [19] up to $100 \mathrm{~Hz}$ and then for frequencies exceeding $1 \mathrm{kHz}$ with a shift factor based on the Williams-Landel-Ferry (WLF) equation [20], or by a direct method called Base Excitation Resonant Mass (BERM) [21, 22] suitable for measuring dynamic properties up to $1000 \mathrm{~Hz}$ [18], etc. However, the laboratory did not have access to these methodologies.

It was also observed that samples' time-dependent relaxation/recovery behaviour may produce density deviations up to $\Delta \rho$ of $0.0006 \%$ (for a resulting hysteresis area of $44 \%$ ) that showed no significance as the uncertainty of density determinations was $\sim 0.0053 \%$.

As planned in the 17RPT02-rhoLiq EMPIR Project the viscoelasticity effect on density results need to be study by a measurement method with a low uncertainty such as the hydrostatic weighing. This may lead to means of comparison that will be able to use the oscillation-type density meters in their maximum metrological capability also with nonNewtonian samples. Or even to know the real limitation of this measuring instrument, to give the most accurate insights for reference documents, such as standards and guides.

Despite not being possible of establish a casual relation between samples' viscoelasticity and density errors, the results of this study gave the information that viscoelastic samples can produce density errors up to $0.18 \%$ (when for the maximum obtained for the high viscosity samples was $0.069 \%$ [8]) with an uncertainty $0.011 \%$ (limited by the uncertainty of the reference density measurement method used).

The EMPIR project "17RPT02-rhoLiq" is carried out with funding of European Union under the EMPIR. The EMPIR is jointly funded by the EMPIR participating countries within EURAMET and the European Union.

\section{References}

1. https://ec.europa.eu/agriculture/sites/agriculture/files/wine/statistics/priceevolution_en.pdf

2. https://ec.europa.eu/agriculture/sites/agriculture/files/olive-oil/prices/marketsituation_en_0.pdf

3. https://www.eea.europa.eu/data-and-maps/indicators/fuel-prices-and-taxes/assessment6

4. ISO 15212-1:1998. Oscillation-type density meters - Part 1: Laboratory instruments

5. ISO 15212-2:2002. Oscillation-type density meters- Part 2: Process instruments for homogeneous liquids

6. OIML G 14: 2011 (E). Density measurement

7. WELMEC Guide 6.4. Guide for packers and importers of e-marked prepacked products

8. A. Furtado, R. Pagel, F. Lorenz, I. Godinho, H. Wolf Annual Trans. of the Nordic Rheol. Soc. 25 (2016)

9. https://www.euramet.org/research-innovation/search-researchprojects/details/?eurametCtcp_project_show $\% 5 B$ project $\% 5 \mathrm{D}=1551$ \&eurametCtcp_pro ject $\% 5$ Bback $\% 5 \mathrm{D}=1081 \& \mathrm{cHash}=515 \mathrm{ddac} 86 \mathrm{e} 38 \mathrm{ea} 4 \mathrm{ac} 36 \mathrm{c} 4 \mathrm{~d} 8 \mathrm{a} 2 \mathrm{e} 0 \mathrm{a} 6969$

10. T. G. Mezger, The Rheology Handbook (E. Coatings, $4^{\text {th }}$ edition, Vincentz Network, 2014)

11. J. G. Savins Rheologica Acta 7(1) 87-93 (1968)

12. K. Al-Malah. Annual Transactions-Nordic Rheology Society 14123 (2006)

13. K. Benyounes, S. Remli, A. Benmounah J. of Physics: Conference Series 1045012008 (2018) 
14. W. Chuin Won, Yong-ik Jin, Misook Kim, Youngseung Lee \& Yoon Hyuk Chang International J. of Food Properties 2012 3076-3089 (2017)

15. JCGM 200:2008. International vocabulary of metrology - Basic and general concepts and associated terms (VIM)

16. ISO 2811-1:2016. Paints and varnishes - Determination of density - Part 1: Pycnometer method

17. JCGM 100:2008. Evaluation of Measurement Data - Guide to the expression of uncertainty in measurement, (GUM 1995 with minor corrections)

18. M. L. Williams, R. F. Landel, J. D. Ferry. J. Am. Chem. Soc. 77 (14): 3701-3707 (1955)

19. ISO 4664-1:2011- Rubber, vulcanized or thermoplastic - Determination of dynamic properties -Part 1: General guidance

20. J.D. Ferry, Viscoelastic Properties of Polymers, John Wiley \& Sons, New York (1980)

21. M. Darlow and E. Zorzi, Mechanical design handbook for elastomers. [the design of elastomer dampers for application in rotating machinery (1981)

22. T. Shoyama and K. Fujimoto, Polymer Testing 67 399-408 (2018) 